\title{
Slow-light enhancement of Beer-Lambert-Bouguer absorption
}

\author{
Mortensen, Asger; Xiao, Sanshui
}

Published in:

Applied Physics Letters

Link to article, DOI:

$10.1063 / 1.2720270$

Publication date:

2007

Document Version

Publisher's PDF, also known as Version of record

Link back to DTU Orbit

Citation (APA):

Mortensen, A., \& Xiao, S. (2007). Slow-light enhancement of Beer-Lambert-Bouguer absorption. Applied Physics Letters, 90(14), 141108. https://doi.org/10.1063/1.2720270

\section{General rights}

Copyright and moral rights for the publications made accessible in the public portal are retained by the authors and/or other copyright owners and it is a condition of accessing publications that users recognise and abide by the legal requirements associated with these rights.

- Users may download and print one copy of any publication from the public portal for the purpose of private study or research.

- You may not further distribute the material or use it for any profit-making activity or commercial gain

- You may freely distribute the URL identifying the publication in the public portal

If you believe that this document breaches copyright please contact us providing details, and we will remove access to the work immediately and investigate your claim. 


\title{
Slow-light enhancement of Beer-Lambert-Bouguer absorption
}

\author{
Niels Asger Mortensen ${ }^{\text {a) }}$ and Sanshui Xiao \\ MIC-Department of Micro and Nanotechnology, Technical University of Denmark, DTU-Building 345 \\ East, DK-2800 Kongens Lyngby, Denmark
}

(Received 16 February 2006; accepted 5 March 2007; published online 5 April 2007)

\begin{abstract}
The authors theoretically show how slow light in an optofluidic environment facilitates enhanced light-matter interactions, by orders of magnitude. The proposed concept provides strong opportunities for improving existing miniaturized chemical absorbance cells for Beer-Lambert-Bouguer absorption measurements widely employed in analytical chemistry. (C) 2007 American Institute of Physics. [DOI: 10.1063/1.2720270]
\end{abstract}

Optical techniques are finding widespread use in chemical and biochemical analysis, and Beer-Lambert-Bouguer (BLB) absorption, in particular, has become one of the classical workhorses in analytical chemistry. ${ }^{1}$ During the past decade, there has been an increasing emphasis on miniaturization of chemical analysis systems ${ }^{2}$ and naturally this has stimulated a large effort in integrating microfluidics ${ }^{3,4}$ and optics in lab-on-a-chip microsystems, ${ }^{5}$ partly defining the emerging field of optofluidics. ${ }^{6,7}$ At the same time, there is an increasing attention to slow-light phenomena as well as the fundamentals and applications of light-matter interactions in electromagnetically strongly dispersive environments. ${ }^{8-12}$ In this letter we consider the classical problem of BLB absorption. As with the phenomenon of photonic band-edge lasing, ${ }^{13}$ we show how slow light in an optofluidic environment facilitates enhanced light-matter interactions, by orders of magnitude, with strong opportunities for improving existing miniaturized chemical absorbance cells.

The principle of a BLB measurement is illustrated in panel (a) of Fig. 1 with an optical probe, with intensity $I_{0}$, incident on a sample with absorption parameter $\alpha_{l}$ due to a concentration of some chemical species. Typically the chemicals will be dissolved in a liquid, but gas and transparent solid phases are in principle also possible. Neglecting coupling issues, the transmitted intensity $I$ will then, quite intuitively, be exponentially damped, $I=I_{0} \exp \left(\gamma \alpha_{l} L\right)$, with $L$ being the optical path length and $\gamma$ being a dimensionless parameter of the slow-light enhanced light-matter interactions. For a uniform medium, of course, we have $\gamma \equiv 1$ and the expression is often referred to as Beer's law. Since $\alpha$ correlates with the concentration of the absorbing chemical species, Beer's law provides optical means for detecting and quantifying the concentration of chemical solutions. ${ }^{1}$ Obviously, the effect relies heavily on having a sufficiently long optical path length and the longer $L$ is the lower a concentration can be monitored for a given sensitivity of the optical equipment measuring $I / I_{0}$. Lab-on-a-chip implementations of chemical absorbance cells are thus facing almost exhausting challenges since the miniaturization, i.e., reduction of $L$, decreases the sensitivity significantly. This problem has already been confirmed experimentally for lab-on-a-chip systems operated in the visible region with $L$ of the order 100 to $1000 \mu \mathrm{m} .{ }^{14}$ In this work, we show a route to achieve enhancement factors $\gamma$ much larger than unity, thus poten-

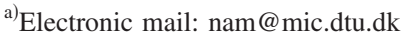

tially compensating for the cost of miniaturization and reduction in optical path length.

In order to explicitly show this phenomenon, we start from the electromagnetic wave equation for the electrical field,

$$
\nabla \times \nabla \times|E\rangle=\epsilon \frac{\omega^{2}}{c^{2}}|E\rangle,
$$

and consider the case of a weakly absorbing medium with $\epsilon=\varepsilon+i \delta \varepsilon$. Since absorption is a weak perturbation, $\delta \varepsilon \ll \varepsilon$, standard first-order electromagnetic perturbation theory is fully adequate to predict the small imaginary shift in frequency, $\Delta \omega \propto i \delta \varepsilon$. For the unperturbed problem, we may obtain the dispersion relation $\omega(\kappa)$ by solving the wave equation (see method section) with $\epsilon=\varepsilon$. For a fixed frequency, the perturbation $i \delta \varepsilon$ changes into an imaginary shift $i \Delta \kappa$ of the wave vector $\kappa$ so that the absorption parameter $\alpha=2 \Delta \kappa$ becomes $\alpha=k\left(c / v_{g}\right)\langle E|\delta \varepsilon| E\rangle /\langle E|\varepsilon| E\rangle$, where the electrical field is the unperturbed field in the absence of absorption, $v_{g}=\partial \omega / \partial \kappa$ is the group velocity, and $k=\omega / c$ is the free-space wave vector. As a reference we consider a homogeneous liquid with $\epsilon_{l}=n_{l}^{2}$ where we have a linear dispersion $\omega(\kappa)$ $=\left(c / n_{l}\right) \kappa$ with a group velocity of $c / n_{l}$ and thus $\alpha_{l}=k \delta \varepsilon / n_{l}$. Next, we imagine that the dispersion is modified by introducing a nonabsorbing (at least compared to the liquid) material of different index in the liquid, see panels (b)-(d) in Fig. 1.

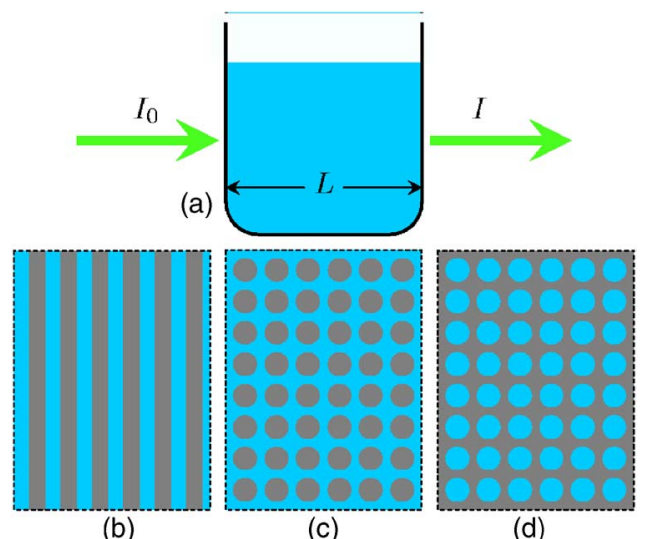

FIG. 1. (Color online) Schematic of (a) classical setup of Beer-LambertBouguer chemical absorbance cell and [(b)-(d)] examples of strongly dispersive environments provided by photonic crystals with dielectric regions (grey) with a dielectric function different from that of the liquid sample (blue). 

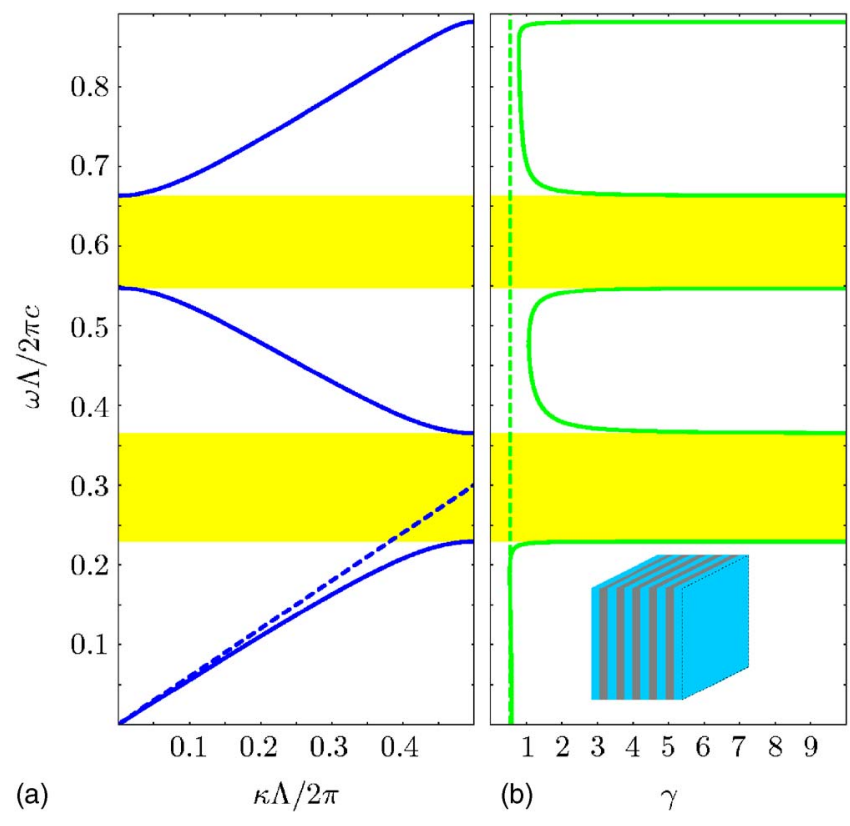

FIG. 2. (Color online) (a) Photonic band structure for normal incidence of either TE or TM polarized light on a Bragg stack of period $\Lambda=a_{l}+a_{2}$ with $n_{l}=1.33, n_{2}=3, a_{l}=0.8 \Lambda$, and $a_{2}=0.2 \Lambda$. Photonic band gaps are indicated by yellow shading and the dashed line indicates the long-wavelength asymptotic limit where $\omega \simeq c \kappa \Lambda /\left(a_{l} n_{l}+a_{2} n_{2}\right)$. (b) Corresponding enhancement factor which peaks and exceeds unity close to the photonic band-gap edges. The dashed line indicates the long-wavelength asymptotic limit where $f \simeq a_{l} n_{l}^{2} /\left(a_{l} n_{l}^{2}+a_{2} n_{2}^{2}\right)$.

Compared to the bare liquid such a composite medium may support an enhancement of the effective absorption. The enhancement factor $\gamma \equiv \alpha / \alpha_{l}$ can now be expressed as

$$
\gamma=f \times \frac{c / n_{l}}{v_{g}}, \quad f \equiv \frac{\langle E \mid D\rangle_{l}}{\langle E \mid D\rangle},
$$

where we have introduced the displacement field $|D\rangle=\varepsilon|E\rangle$. The integral in the nominator of the filling factor $0<f<1$ is restricted to the region containing the absorbing fluid while the integral in the denominator is spatially unrestricted. This expression clearly demonstrates how BLB absorption benefits from slow-light phenomena. For liquid infiltrated photonic crystals and photonic crystal waveguides, it is possible to achieve $v_{g} \ll c$ and at the same time have a filling factor of the order unity, $f \sim 1$, whereby significant enhancement factors become feasible. The effective enhancement of the absorption can also be understood in terms of an effective enhancement of the light-matter interaction time given by the Wigner-Smith delay time $\tau$. For the homogeneous problem, we have $\tau_{l} \sim L /\left(c / n_{l}\right)$ while for the strongly dispersive problem $\tau \sim L / v_{g}$ so that $\gamma \sim \tau / \tau_{l} \propto\left(c / n_{l}\right) / v_{g}$ in agreement with the result in Eq. (2) rigorously derived from perturbation theory. The presence of the filling factor $f$ is also easily understood since only the fraction $f$ of the light residing in the fluid can be subject to absorption. These conclusions may also be extended to nonperiodic systems, including enhanced absorption in disordered systems as well as intracavity absorbance configurations, by use of scattering matrix arguments. 15

Let us next illustrate the slow-light enhancement for the simplest possible structure: a Bragg stack with normal incidence of electromagnetic radiation. Panel (a) of Fig. 2 shows the photonic band structure of an optofluidic Bragg

Downloaded 17 Jun 2010 to 192.38.67.112. Redistribution subject
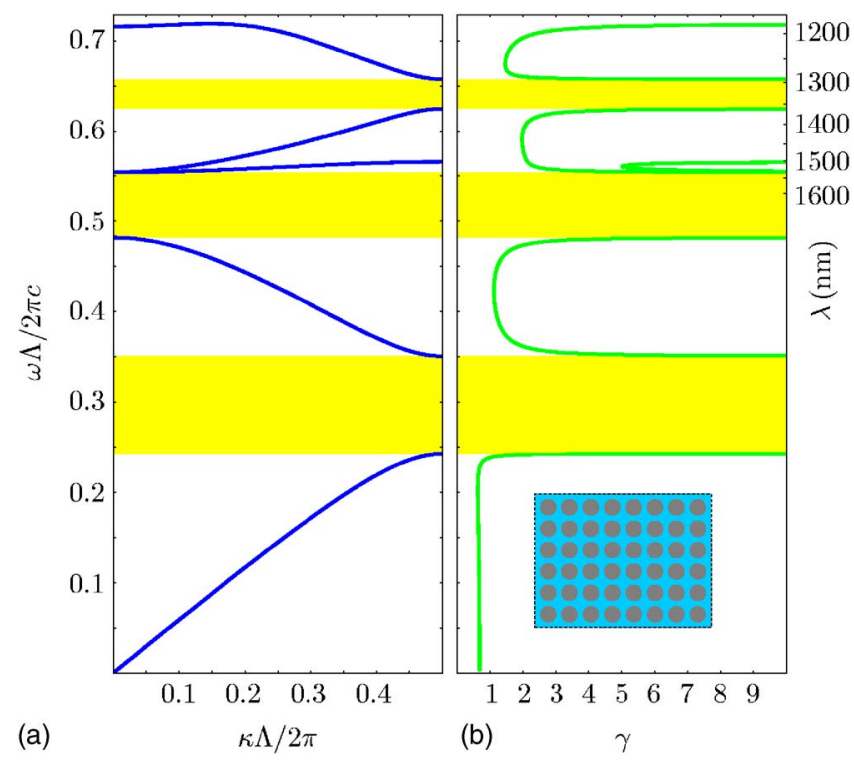

FIG. 3. (Color online) (a) Photonic band structure for propagation of TM polarized light along the $\Gamma X$ direction in a square lattice of period $\Lambda$ with dielectric rods of diameter $d=0.4 \Lambda$ and $\varepsilon=10.5$. Photonic band gaps are indicated by yellow shading. (b) Corresponding enhancement factor which exceeds unity for the flat bands in general and the third band in particular. The right $y$ axis shows the results in terms of the free-space wavelength when results are scaled to a structure with $\Lambda=850 \mathrm{~nm}$.

stack of period $\Lambda=a_{l}+a_{2}$ with the low-index material layers of width $a_{l}=0.8 \Lambda$ being a liquid with refractive index $n_{l}=1.33$ while the high-index layers have a width $a_{2}=0.2 \Lambda$ and a refractive index $n_{2}=3$. Photonic band gaps are indicated by yellow shading and the dashed line indicates the long-wavelength asymptotic limit where the Bragg stack has a metamaterial response with a close-to-linear dispersion $\omega \simeq c \kappa \Lambda /\left(a_{l} n_{l}+a_{2} n_{2}\right)$. When approaching the band-gap edges, the dispersion flattens corresponding to a slow group velocity. It is well known that the flat dispersion originates from a spatial localization of the field onto the high-index layers and thus $f \ll 1$ near the band edges where the inverse group velocity diverges. However, in spite of the localization, the enhancement factor may still exceed unity as shown in panel (b) where the dashed line indicates the long-wavelength asymptotic limit with $f \simeq\langle 1|\epsilon| 1\rangle_{l} /\langle 1|\epsilon| 1\rangle$ $=a_{l} n_{l}^{2} /\left(a_{l} n_{l}^{2}+a_{2} n_{2}^{2}\right)$. In order to further benefit from the slowlight enhanced light-matter interaction, we obviously have to pursue optofluidic structures supporting both low group velocity and at the same time large filling factors. Figure 3 shows one such example where high-index dielectric rods are arranged in a square lattice. Compared to the Bragg stack, some of the modes in this structure have both a low group velocity and at the same time a reasonable value of the filling factor $f$. Particularly the third band in panel (a) is quite flat and with a finite $f$ giving rise to an enhancement factor $\gamma$ exceeding 5 even at the center of the band. As indicated on the right $y$ axis, the enhancement may have a bandwidth of order of $50 \mathrm{~nm}$ for a pitch around $\Lambda \sim 850 \mathrm{~nm}$, which indeed makes fabrication of such structures realistic with state of the art micro- and nanofabrication facilities. As a final example, Fig. 4 shows the result of introducing a line-defect waveguide in such a structure. The waveguide mode has $f \sim 60 \%$ combined with a low group velocity near the band edges.

AIP license or copyright; see http://apl.aip.org/apl/copyright.jsp 


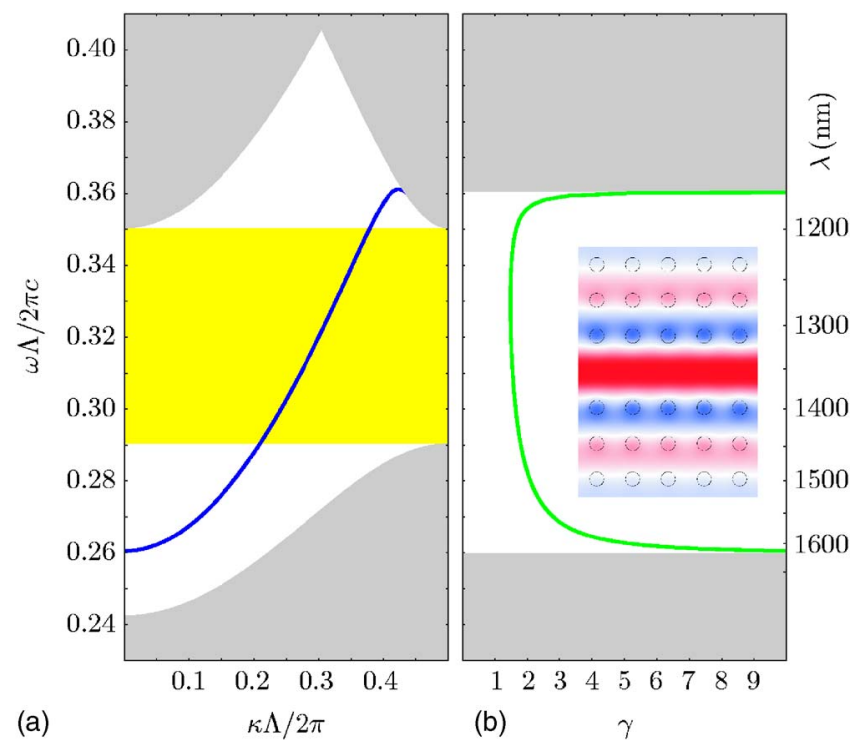

FIG. 4. (Color online) (a) Photonic band structure for propagation of TM polarized light along the $\Gamma X$ direction in a line-defect waveguide in a square lattice of period $\Lambda$ with dielectric rods of diameter $d=0.4 \Lambda$ and $\varepsilon=10.5$. The complete photonic band gap of the photonic crystal is indicated by yellow shading while grey shading indicates the finite density of states in the photonic crystal due to the projected bands in the Brillouin zone. (b) Corresponding enhancement factor which exceeds unity over the entire bandwidth. The right $y$ axis shows the results in terms of the free-space wavelength when results are scaled to a structure with $\Lambda=420 \mathrm{~nm}$. The inset shows the electrical field of the waveguide mode at the $\Gamma$ point $\kappa=0$.

For the above numerical results, fully vectorial eigenmodes of Maxwell's equations, Eq. (1), with periodic boundary conditions computed by preconditioned conjugategradient minimization of the block Rayleigh quotient in a planewave basis, using a freely available software package. ${ }^{16}$ For the resolution, we have used a basis of $2^{10}$ plane waves for the one-dimensional problem in Fig. 2 and $2^{10} \times 2^{10}$ plane waves for the two-dimensional problem in Fig. 3. In Fig. 4 we have used a resolution of $2^{7} \times 2^{7}$ and a supercell of size $1 \times 7$.

In the above examples we have for simplicity considered dielectric constants corresponding to semiconductor materials suitable for the near-infrared regime. However, we would like to emphasize that applications exist also in the visible, midinfrared, far-infrared, and even the microwave and subterahertz regimes. The predicted enhancement of light-matter interactions makes liquid-infiltrated photonic crystals obvious candidates for improving existing miniaturized chemical absorbance cells. Previous work on liquid-infiltrated photo- nic crystals ${ }^{17-21}$ has focused on the solid type with liquidinfiltrated voids illustrated in panel (d) of Fig. 1, while we in this work have focused on rod-type photonic crystals which have the technological strong advantage that they are permeable to an in-plane liquid flow, thus making them integrable with microfluidic channels in plane lab-on-a-chip technology.

In conclusion, we have studied the potential of using liquid-infiltrated photonic crystals to enhance Beer-LambertBouguer absorption. The slow-light enhancement of the absorption, by possibly orders of magnitude, may be traded for yet smaller miniaturized systems or for increased sensitivity of existing devices.

This work is financially supported by the Danish Council for Strategic Research through the Strategic Program for Young Researchers (Grant No. 2117-05-0037).

${ }^{1}$ D. A. Skoog, D. M. West, and F. J. Holler, Fundamentals of Analytical Chemistry (Saunders College Publishing, New York, 1997).

${ }^{2}$ D. Janasek, J. Franzke, and A. Manz, Nature (London) 442, 374 (2006).

${ }^{3}$ T. M. Squires and S. R. Quake, Rev. Mod. Phys. 77, 977 (2005).

${ }^{4}$ G. M. Whitesides, Nature (London) 442, 368 (2006).

${ }^{5}$ E. Verpoorte, Lab Chip 3, 42N (2003).

${ }^{6}$ D. Psaltis, S. R. Quake, and C. H. Yang, Nature (London) 442, 381 (2006).

${ }^{7}$ C. Monat, P. Domachuk, and B. J. Eggleton, Nature Photonics 1, 106 (2007).

${ }^{8}$ P. Lodahl, A. F. van Driel, I. S. Nikolaev, A. Irman, K. Overgaag, D. L. Vanmaekelbergh, and W. L. Vos, Nature (London) 430, 654 (2004).

${ }^{9}$ M. Soljacic and J. D. Joannopoulos, Nat. Mater. 3, 211 (2004).

${ }^{10}$ Y. A. Vlasov, M. O'boyle, H. F. Hamann, and S. J. McNab, Nature (London) 438, 65 (2005).

${ }^{11}$ R. S. Jacobsen, K. N. Andersen, P. I. Borel, J. Fage-Pedersen, L. H. Frandsen, O. Hansen, M. Kristensen, A. V. Lavrinenko, G. Moulin, H. Ou, C. Peucheret, B. Zsigri, and A. Bjarklev, Nature (London) 441, 199 (2006).

${ }^{12}$ S. Noda, Science 314, 260 (2006).

${ }^{13}$ J. P. Dowling, M. Scalora, M. J. Bloemer, and C. M. Bowden, J. Appl. Phys. 75, 1896 (1994).

${ }^{14}$ K. B. Mogensen, J. El-Ali, A. Wolff, and J. P. Kutter, Appl. Opt. 42, 4072 (2003).

${ }^{15}$ C. W. J. Beenakker and P. W. Brouwer, Physica E (Amsterdam) 9, 463 (2001).

${ }^{16}$ S. G. Johnson and J. D. Joannopoulos, Opt. Express 8, 173 (2001).

${ }^{17}$ M. Lončar, A. Scherer, and Y. M. Qiu, Appl. Phys. Lett. 82, 4648 (2003).

${ }^{18}$ E. Chow, A. Grot, L. W. Mirkarimi, M. Sigalas, and G. Girolami, Opt. Lett. 29, 1093 (2004).

${ }^{19}$ H. Kurt and D. S. Citrin, Appl. Phys. Lett. 87, 241119 (2005).

${ }^{20}$ D. Erickson, T. Rockwood, T. Emery, A. Scherer, and D. Psaltis, Opt. Lett. 31, 59 (2006).

${ }^{21}$ T. Hasek, H. Kurt, D. S. Citrin, and M. Koch, Appl. Phys. Lett. 89, 173508 (2006). 